\title{
Primary Multiple Mesenteric Liposarcoma of the Transverse Mesocolon
}

\author{
Sandeep Sachidananda, Arunkumar Krishnan ${ }^{1}$, Raja Ramesh, Sivaprakasam Kuppurao \\ Departments of General Surgery and ${ }^{1}$ Gastroenterology and Hepatology, Stanley Medical College and Hospital, Chennai, India
}

Liposarcomas are common tumors arising in the retroperitoneum. However, a primary mesenteric liposarcoma is a rare entity and less than 50 cases have been reported so far. Further, a liposarcoma arising in the transverse mesocolon is very unusual, and cases of multiple primary tumors arising from the transverse mesocolon are extremely rare. We want to report a case of a multiple primary mesenteric liposarcoma arising from the transverse mesocolon in a 63-year-old female who was successfully treated by surgery. Because a primary mesenteric liposarcoma is a rare entity, it should be considered with the differential diagnosis of an abdominal mesenchymal tumor. It can be diagnosed preoperatively by using contrast enhanced computed tomography and magnetic resonance imaging. The treatment for such a liposarcoma is surgical resection with sufficient surgical margin; the role of adjuvant therapy has yet to be defined.

Keywords: Liposarcoma; Mesentric; Transverse magacolon; Diagnosis

\section{INTRODUCTION}

Liposarcomas are the second most common type of soft tissue sarcomas [1]. They are commonly known to occur in the retroperitoneum. A primary mesenteric origin of a liposarcoma is an unusual occurrence and has been reported to occur from the small bowel mesentery. A primary origin from the transverse mesocolon is a rare occurrence $[2,3]$. The multiplicity of these tumors arising from the transverse mesocolon in our case makes it a unique one.

\section{CASE REPORT}

A 63-year-old female with no comorbidities presented with gradually progressive, painless, upper abdominal distension and loss

Received: July 17, 2012 - Accepted: October 3, 2012

Correspondence to: Arunkumar Krishnan, M.D.

Department of Gastroenterology and Hepatology, Stanley Medical College and Hospital, C-137, Karthikeyan Salai, Periyar Nagar, Perambur, Chennai 600082, Tamilnadu State, India

Tel: +91-44-25289595, Fax: +91-442-5289595

E-mail: dr.arunkumarpillai@gmail.com.

(C) 2013 The Korean Society of Coloproctology

This is an open-access article distributed under the terms of the Creative Commons Attribution NonCommercial License (http://creativecommons.org/licenses/by-nc/3.0) which permits unrestricted noncommercial use, distribution, and reproduction in any medium, provided the original work is properly cited. of appetite for one year. Physical examination revealed a distended abdomen and epigastrium, as well as right and left hypochondrium fullness extending to the umbilicus. A nontender mass of about $15 \mathrm{~cm} \times 12 \mathrm{~cm}$ in size was palpable in the epigastrium, the right and the left hypochondrium, and the umbilical regions. The mass was firm, had a smooth surface, moved from side to side, and fell forward when the patient was in the knee-elbow position. The findings of abdominopelvic ultrasound showed a $12.5 \mathrm{~cm} \times$ $9.6 \mathrm{~cm}$ uniformly echogenic lesion with unclear margins in the midline; the lesion occupied the epigastric and umbilical regions and pushed bowel loops to the periphery.

Contrast enhanced computed tomography (CECT) of the abdomen and the pelvis showed a space-occupying lesion in the epigastric/umbilical region measuring $16 \mathrm{~cm} \times 16 \mathrm{~cm} \times 10 \mathrm{~cm}$. The lesion was a nonhomogenous mass with irregular enhancement and displaced the bowel loops (straddling sign, Fig. 1). Bowel loops were located between the tumor and the anterior abdominal wall, suggesting a probable mesenteric origin [4]. No evidence of a retroperitoneal lymphadenopathy was found, and the lesion was reported as a large teratoma occupying the anterior epigastric/umbilical regions with a possible mesenteric origin.

The patient underwent surgery. A midline laparotomy was done, and the peritoneal cavity was explored. A huge lipomatous mass measuring $23 \mathrm{~cm} \times 16 \mathrm{~cm} \times 12 \mathrm{~cm}$ was found in the lesser sac over the transverse mesocolon. The lesser sac was opened, and the mass was found to be well defined without any major vascular 


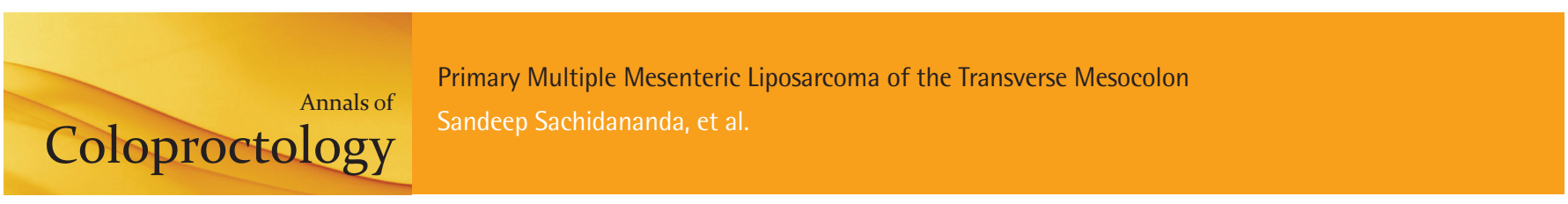

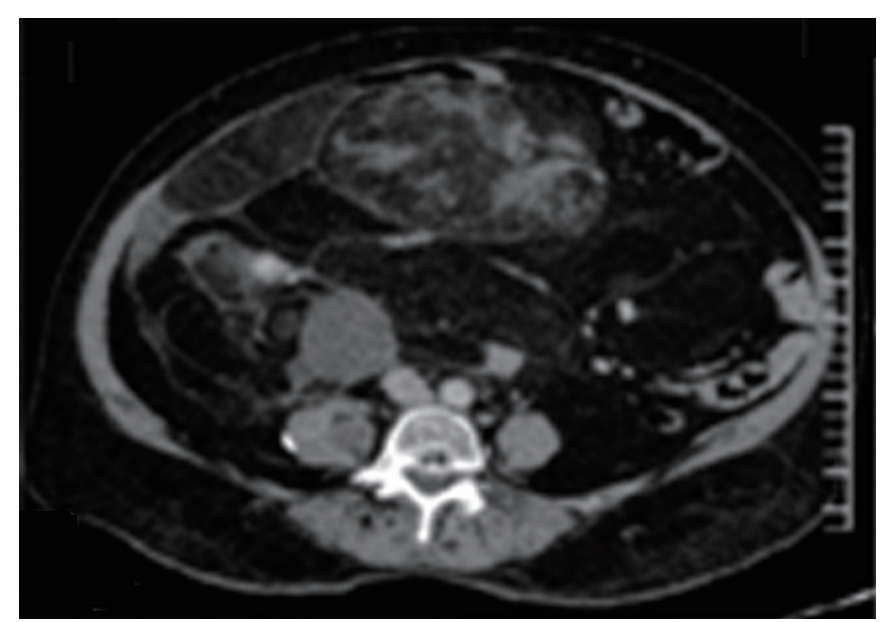

Fig. 1. Contrast enhanced computed tomography of the abdomen showing a large tumor with nonhomogenous, irregular enhancement and the presence of a bowel loop between the tumor and the anterior abdominal wall (straddling sign).

invasion. The tumor was excised in toto with macroscopically negative margins. On further exploration, multiple such separated masses with sizes ranging from 4 to $7 \mathrm{~cm}$ were found to be attached to the transverse mesocolon (Figs. 2 and 3), and each was excised with clear margins. Postoperative period was uneventful. Histopathology showed similar features suggestive of an atypical lipoma or a well-differentiated liposarcoma in all of the excised lesions, suggesting possible multicentricity or the presence of satellite nodules.

\section{DISCUSSION}

The liposarcoma is the second most common soft tissue sarcoma and frequently occurs in the lower extremities and the retroperitoneum. Primary tumors of the mesentery are uncommon. Less than 50 such cases of a primary mesenteric liposarcoma have been reported to date, and very few cases of a liposarcoma of the transverse mesocolon have been reported $[2,3]$. A mesenteric liposarcoma is a tumor seen in adults aged 50 to 70 years and is slightly more common in males [5]. It is usually nonmetastasizing and locally aggressive, presents as a deep-seated mass located more towards the root of the mesentery rather than near the intestine, and can grow to a large size. It is often painless and presents with nonspecific vague symptoms like anorexia, early satiety, and abdominal distention, and occasionally can cause subacute intestinal obstruction or volvulus. The main differential diagnosis for mesenteric tumors is a malignant lymphoma, leiomyosarcoma, or pelvic lipomatosis/lipodystrophy [5].

A liposarcoma is histologically defined as a tumor composed of lipoblasts and can secrete substances such as granulocyte-colonystimulating factor (G-CSF) [3]. For diagnosis, computed tomographic (CT) scans and magnetic resonance imaging (MRI) can

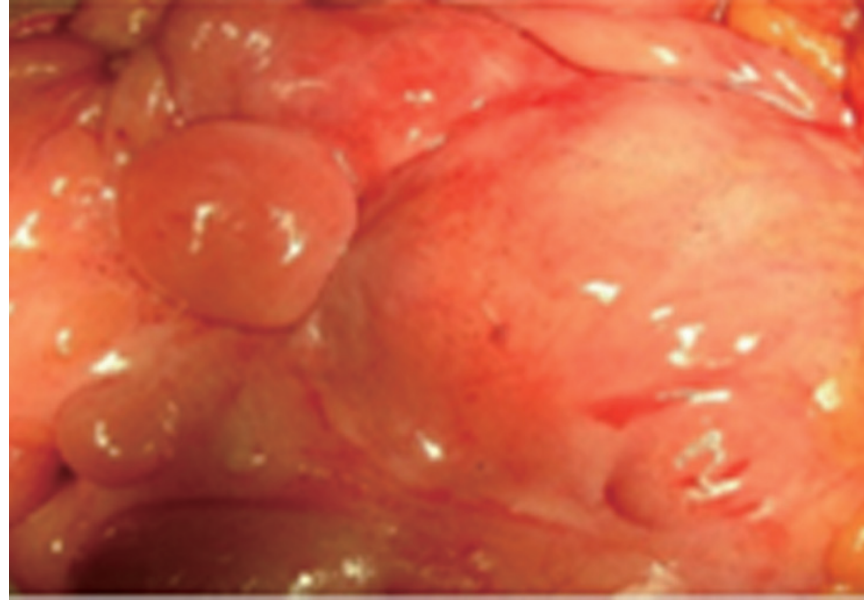

Fig. 2. Multiple lipomatous tumors arising from transverse mesocolon.

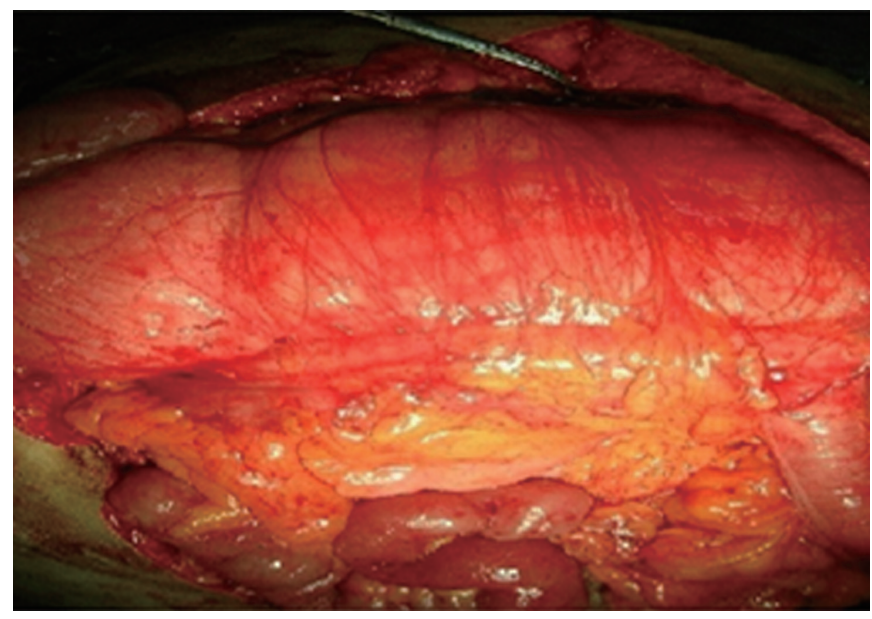

Fig. 3. Intraoperative picture showing a large tumor arising from the transverse mesocolon.

provide information regarding the important structures, as well as the tissue characteristics, of the tumor. The characteristics of a liposarcoma on CT images are nonhomogeneity, infiltration or poor marginalization, and higher CT contrast enhancement than normal fats. The enhancement on CT changes according to the histological grade [1]. In the present case, CT scans revealed a tumor with nonhomogeneous, irregular enhancement. The treatment strategy for a mesenteric liposarcoma is surgical resection with sufficient surgical margin. Complete surgical resection at the time of primary presentation is likely to improve the chance for long-term survival, as well as distant recurrence-free survival, and positive surgical margins are the main predictors of local relapse [5]. The roles of preoperative and postoperative adjuvant chemotherapy and radiotherapy are not very clear. Although reports advocate their usefulness, their survival benefits in the case of a liposarcoma has yet to be established. The key chemotherapy drug is 
doxorubicin while Ifosfamide, dacarbazine and cisplatin have also been used [4].

In conclusion, although a primary mesenteric liposarcoma is a rare entity, it should be considered in the differential diagnosis of abdominal mesenchymal tumors. It usually presents as a slowgrowing tumor that can attain large sizes and cause intestinal obstruction. It can be diagnosed preoperatively by using CECT and MRI. The treatment of choice for such a liposarcoma is surgical resection with sufficient surgical margin, but the role of adjuvant therapy has yet to be defined.

\section{CONFLICT OF INTEREST}

No potential conflict of interest relevant to this article was reported.

\section{REFERENCES}

1. Hirakoba M, Kume K, Yamasaki M, Kanda K, Yoshikawa I, Otsuki M. Primary mesenteric liposarcoma successfully diagnosed by preoperative imaging studies. Intern Med 2007;46:373-5.

2. Yoshiyuki M, Kazuhiko Ne, Tomoo O, Yoshihiro N, Fujio W, Takeshi K. A case of primary liposarcoma arisen from the mesentery of the transverse colon. J Jpn Surg Assoc 2003;64:2221-4.

3. Nakamura A, Tanaka S, Takayama H, Sakamoto M, Ishii H, Kusano $\mathrm{M}$, et al. A mesenteric liposarcoma with production of granulocyte colony-stimulating factor. Intern Med 1998;37:884-90.

4. Ishiguro S, Yamamoto S, Chuman H, Moriya Y. A case of resected huge ileocolonic mesenteric liposarcoma which responded to pre-operative chemotherapy using doxorubicin, cisplatin and ifosfamide. Jpn J Clin Oncol 2006;36:735-8.

5. Burgohain J, Kathiresan N, Satheesan B. Dumbbell-shaped mesenteric liposarcoma: a case report with review of the literature. Internet J Surg 2008;15(2) [cited 2013 Jun 1]: [about 2 p.]. Available from: http://archive.ispub.com/journal/the-internet-journalof-surgery/volume-15-number-2/dumbbell-shaped-mesentericliposarcoma-a-case-report-with-review-of-the-literature.html\# sthash.3W4jux8m.dpbs. 